\title{
Parametric Study For Rectangular Microstrip Patch Antennas
}

\author{
S. S. Yavalkar ${ }^{1}$, R. T. Dahatonde ${ }^{2}$, Dr. S. S. Rathod ${ }^{3}$, Dr. S. B. Deosrkar ${ }^{4}$ \\ ${ }^{1,3}$ Sardar Patel Inst. Of Technology, Mumbai, India \\ ${ }^{2}$ Sardar Patel College of Engg., Mumbai,India \\ ${ }^{4}$ Principal, VPCOE, Baramati, India
}

\begin{abstract}
This paper discusses the parametric study of Microstrip Patch Antenna. A simple Rectangular Microstrip Patch Antenna (RMSA) was designed and simulated using Zeland's Method of Moment (MoM) based EM simulation package IE3D and its various parameters such as return loss, VSWR and input impédance were obtained. Then effect of change of width, lenght, loss tangent, feed location, etc is observe on above parameters. Keywords-Rectangular Microstrip Antenna, Modified Shapes, BW Enhancement, Antenna Design.
\end{abstract}

\section{INTRODUCTION}

Physically, MSA consists of a metallic radiating patch backed up by a dielectric substrate and a ground plane below that. These days, MSAs are widely used in many applications due to their inherent advantages such as low profile, lightweight, planer configuration and ease of fabrication. However, main limitation of MSAs is their inherently narrow BW, typically about $5 \%$ relative to resonant frequency [1].

Most of the Wireless communication applications need antenna with broad BW. Therefore, most of the recent research activities in MSA are aimed towards development of MSAs with wide impedance BW without sacrificing return loss of the antenna. Many such techniques are proposed in literature. Most of these techniques are summarized in [2].

However, techniques such as use of an thick substrate also introduces a large inductance due to increase length of the probe feed, resulting in a maximum BW of less than $10 \%$ of the resonant frequency. Also, though the designs consisting of stacked patches yields higher BWs (10\% to $20 \%$ of the resonant frequency); these designs are complex for fabrication. BW of MSA can also be increased by cutting a resonant slot inside the patch or by using multi resonator gap coupled and stacked configurations [2]. In this paper, these techniques are developed to enhance BW of RMSA. A comparative analysis of the various geometries of MSA obtained by cutting slots inside the radiating patch indicate considerable improvement in $\mathrm{BW}$ without much sacrifice on other performance parameters of MSA such as return loss, VSWR and its input impedance.

\section{A. Design of RMSA}

\section{DESIGN OF PATCH ANTENNA}

According to Transmission line model, MSA is represented as two slots separated by a transmission line. The Microstrip separates two dielectrics, i.e. air and substrate. Hence most of the electric field lines reside inside the substrate and some extend to air. This transmission line cannot support pure TEM mode of propagation since the phase velocities would be different in air and the substrate. Hence, effective dielectric constant must be obtained in order to account for fringing fields. The value of effective dielectric constant is less than dielectric constant of the substrate, because the fringing fields around the periphery of the patch are not confined in the dielectric substrate, but are also spread in the air. The value of this effective dielectric constant is given by [4];

$$
\varepsilon_{e f f}=\frac{\left(\varepsilon_{r}+1\right)}{2}+\frac{\left(\varepsilon_{r}-1\right)}{2}\left[1+\frac{12 h}{W}\right]^{-\frac{1}{2}}
$$

Where in $\varepsilon_{\text {eff }}$ is effective dielectric constant and $\varepsilon_{r}\left(\mathrm{Fm}^{-1}\right), h(\mathrm{~mm}), W(\mathrm{~mm})$ are represents dielectric constant, height and width of the substrate respectively.

For RMSA to be an effective radiator, $\mathrm{W}$ should be taken equal to a half wavelength corresponding to the two dielectric mediums (i.e. substrate and air) [1].

$$
W=\frac{c}{2 f_{0} \sqrt{\frac{\varepsilon_{r}+1}{2}}}
$$

Where, $\mathrm{c}$ is velocity of light in free space. 
The fringing fields along the width can be modeled as radiating slots increasing electrical length of patch than physical length [3]. This increase in length is given as;

$$
\Delta L=0.412 h\left[\frac{\left(\varepsilon_{e f f}+0.3\right)\left(\frac{W}{h}+0.264\right)}{\left(\varepsilon_{e f f}-0.258\right)\left(\frac{W}{h}+0.813\right)}\right]
$$

Thus at resonance frequency, effective length of the patch is;

$L_{e}=L+2 \Delta L$

From these equations, dimensions were obtained for $1.57 \mathrm{GHz}$ of $\mathrm{L}$ band, The length and width of the RMSA was found to be $55 \mathrm{~mm}$ and $45.5 \mathrm{~mm}$, respectively. Fig. 1 shows above geometry.

The geometry of this RMSA is shown in Fig.1. This design was simulated using Zeland's MoM based EM Simulation Package, IE3D. For simulation purpose FR4 substrate with dielectric constant of 4.47 with thickness of $1.6 \mathrm{~mm}$ was considered. The patch was fed by a $50 \Omega$ coaxial feed line.

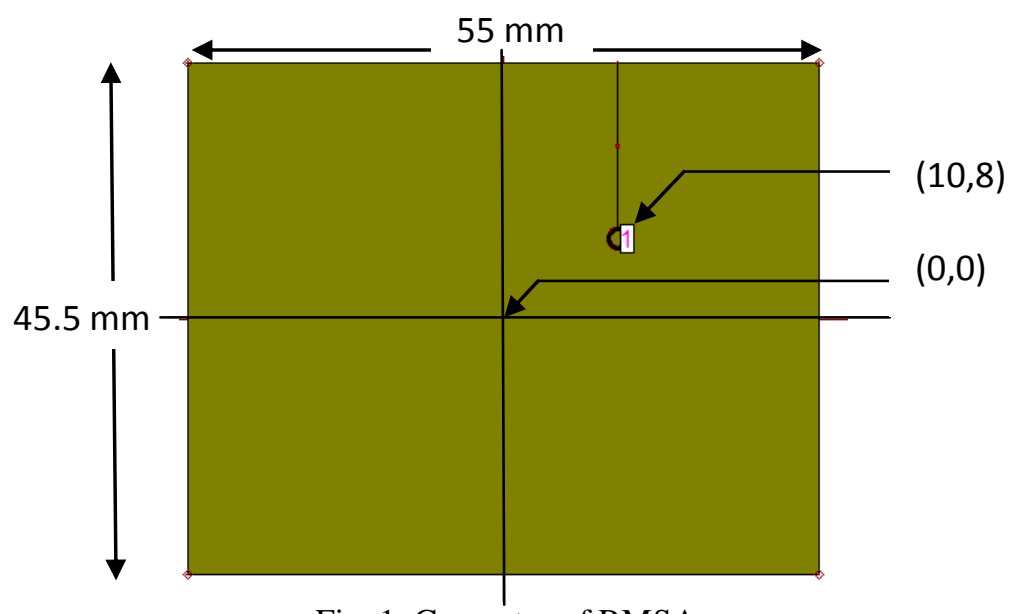

\section{B. Effect of Feed Location}

Fig. 1. Geometry of RMSA

The feed-point should be located at a point on patch where input impedance is 50 at resonance frequency. Return loss at different locations on the patch is compared and a point where return loss (RL) is most negative is selected as a feed-point. The centre of the patch is taken at origin and feed-point location is given by coordinates (Xf, Yf) with respect to origin. There exists a point along length where RL is minimum [1].Fig.2 shows the return loss plot. The feed point at $(10,8)$ giving most negative RL while from smith chart feed at $(10,8)$ giving $50 \Omega$ of impedance.

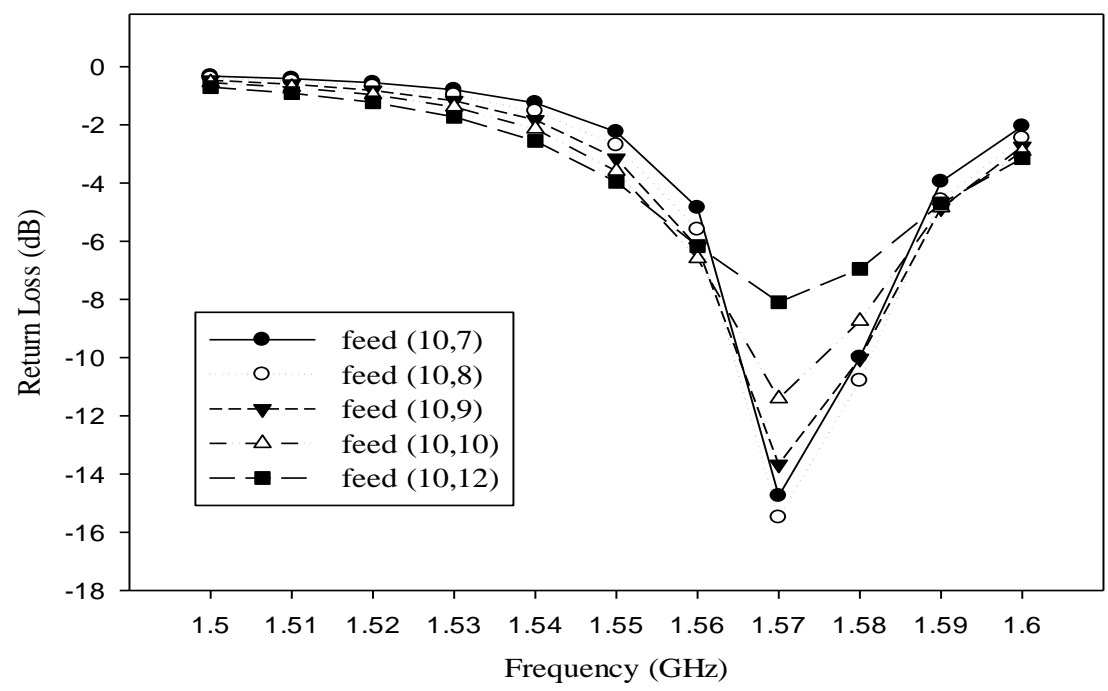

Fig.2. Effect of Feed Location on Return Loss 


\section{Effect of Height}

The RL plots for two different values of $\mathrm{h}(15 \mathrm{~mm}$ and $3 \mathrm{~mm})$ are shown in Fig. 5 for $\mathrm{L}=55 \mathrm{~mm}, \mathrm{~W}=44.5 \mathrm{~mm}$, $\varepsilon r=4.4$ and feed at $(10,08)$. Fig. 3 shows the effect of height on return loss. With an increase in $\mathrm{h}$ from $1.5 \mathrm{~mm}$ to $3 \mathrm{~mm}$, following effects are observed:

- With the increase in $\mathrm{h}$, the fringing fields from the edges increase, which increases the extension in length $\mathrm{L}$ and hence the effective length, thereby decreasing the resonance frequency.

- On the other hand with the increase in $\mathrm{h}$, the $\mathrm{W} / \mathrm{h}$ ratio reduces which decreases e and hence increases the resonance frequency. However, the effect of the increase in $\Delta \mathrm{L}$ is dominant over the decrease in $\varepsilon r$. Therefore, the net effect is to decrease the resonance frequency.

- The input impedance plot moves clockwise (i.e., an inductive shift occurs) due to the increase in the probe inductance of the coaxial feed.

- The BW of the antenna increases. However, for the thicker substrate, this BW is not the maximum [1].

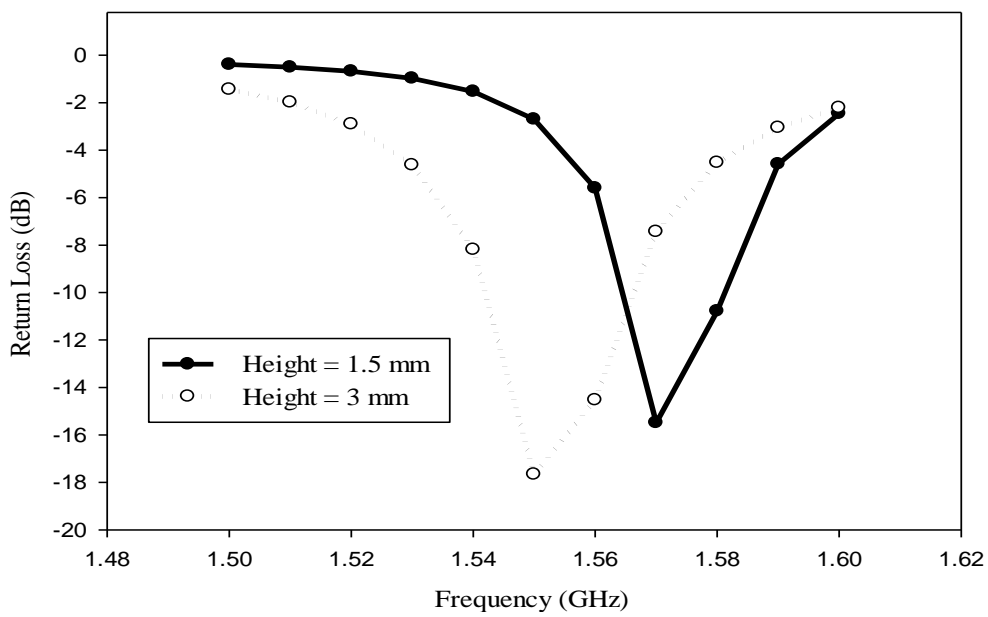

Fig.3. Effect of Height on Return Loss

\section{Effect of Width}

With an increase in $\mathrm{W}$ from $43.5 \mathrm{~mm}$ to $44.5 \mathrm{~mm}$, the following effects are observed:

- The resonance frequency decreases from $1.64 \mathrm{GHz}$ to $1.57 \mathrm{GHz}$ due to the increase in $\Delta \mathrm{L}$ and $\varepsilon$ r.

- The BW of the antenna increases; however, it is not very evident from these plots, because the feed point is not optimum for the different widths. Accordingly, a better comparison will be obtained when the feed point is optimized for the individual widths [1].

Fig.4 shows the effect on width on return loss and bandwidth.

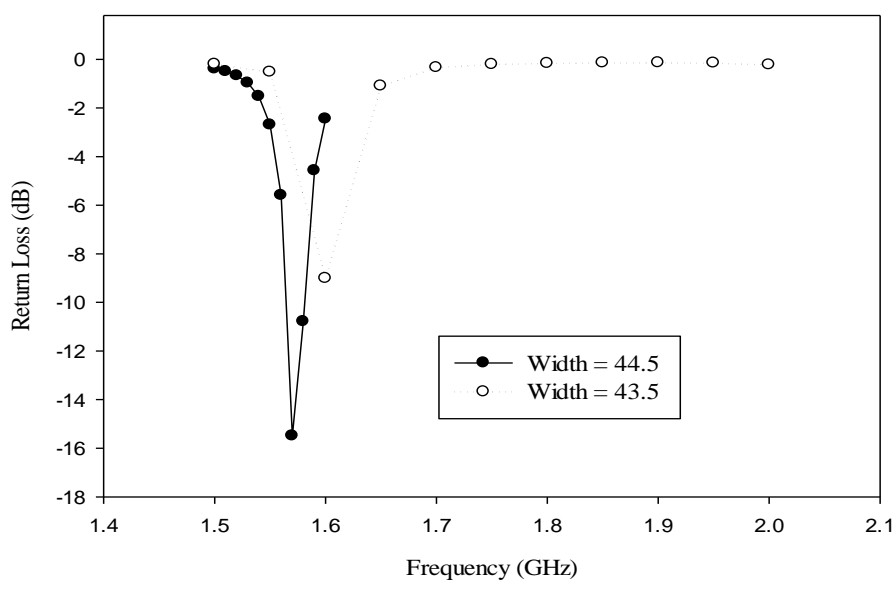

Fig.4. Effect of Width on Return Loss 


\section{E. Effect of $\square \mathbf{r}$}

For RMSA with $\mathrm{L}=55 \mathrm{~mm}, \mathrm{~W}=44.5 \mathrm{~mm}$ and feed at $(10,08)$, when $\varepsilon \mathrm{r}$ is decreased to 1 , the resonance frequency increases. A better comparison of effect of $\mathrm{\varepsilon r}$ is obtained when the antenna is designed to operate in the same frequency range for different values of er [1]. Fig.5 shows effect $\varepsilon r$ on return loss and bandwidth.

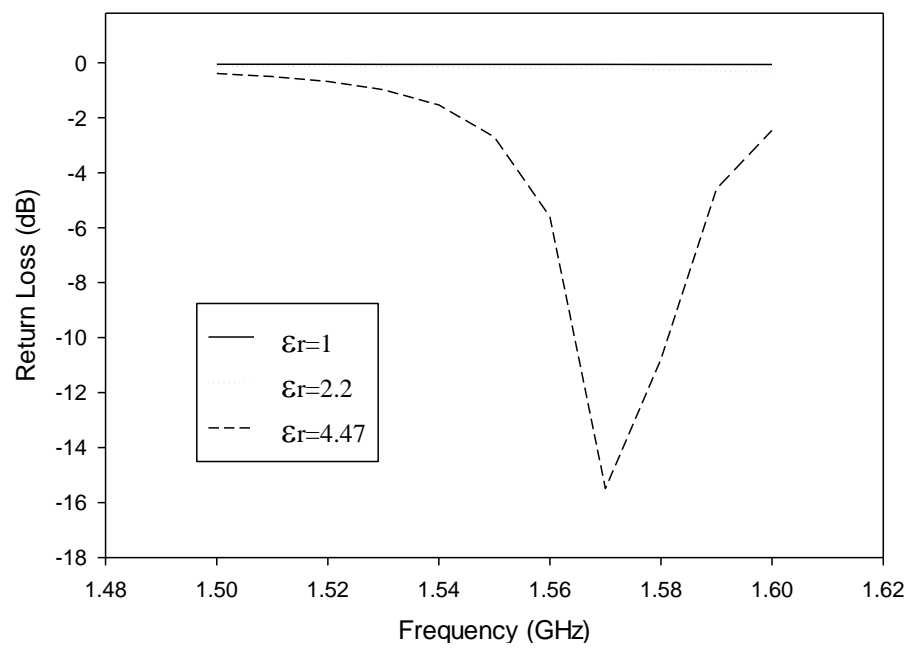

Fig.5. Effect of $\varepsilon r$ on Return Loss

\section{F. Effect of loss tangent $(\tan \delta)$}

For RMSA with $\mathrm{L}=55 \mathrm{~mm}, \mathrm{~W}=44.5 \mathrm{~mm}$ and feed at $(10,08)$, when tan $\delta$ is decreased from 0.01 to 0.001 the resonance frequency decreases. Also it is found that lesser the loss tangent less the loss in probe giving the wider bandwidth. Fig.6 shows effect of loss tangent on return loss and bandwidth.

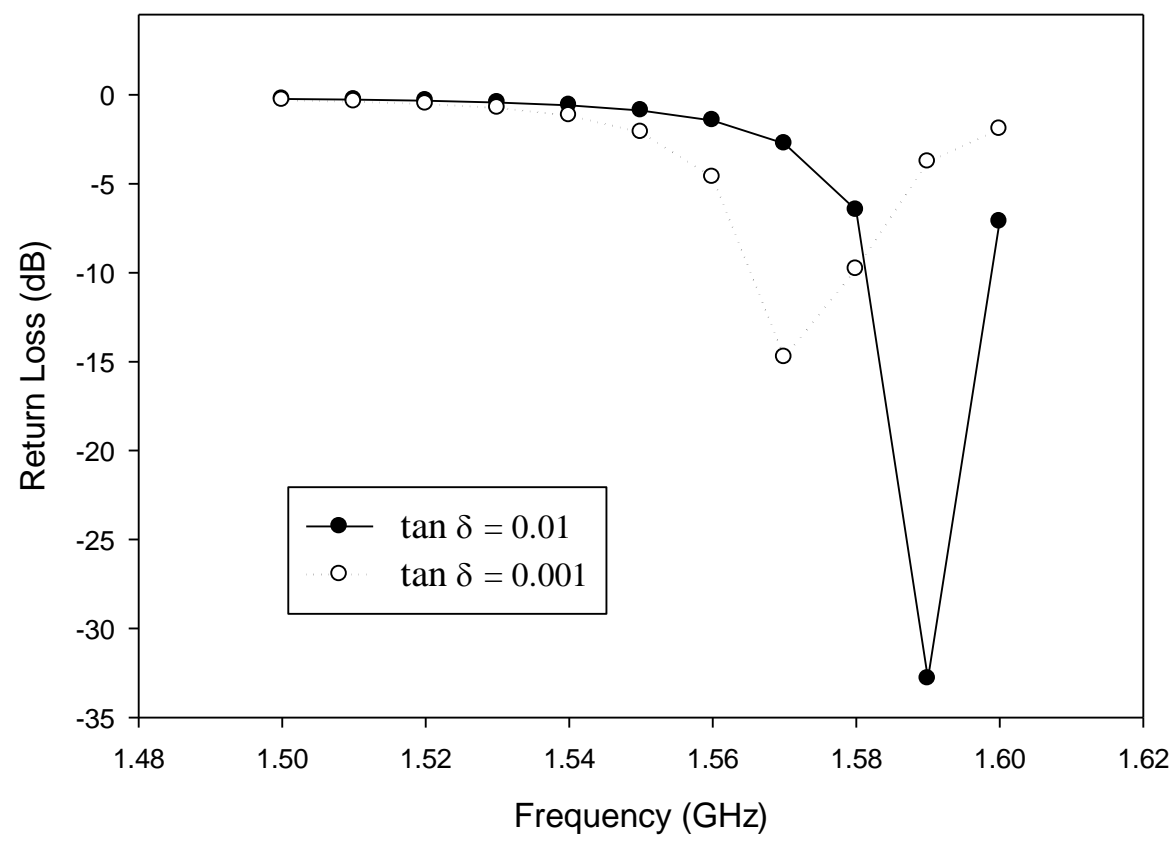

Fig.6. Effect of loss tangent on return loss

The table below, showing the above comparisons in a tabular format so that we can conclude the effect of parameters on resonance frequency and bandwidth. 


\begin{tabular}{|l|l|l|}
\hline Parameters & Resonance Frequency & Bandwidth \\
\hline Feed Location & No shift & --- \\
\hline Height & Increases & Increases \\
\hline Width & Decreases & Decreases \\
\hline$\varepsilon r$ & Decreases & Decreases \\
\hline Loss Tangent & Increases & Decreases \\
\hline
\end{tabular}

\section{CONCLUSION}

A comparative study of effect various parameters on bandwidth and return loss of patch antenna is presented in this paper. It was observed that, to increases resonance frequency height and loss tangent is to be increased whereas width and $\varepsilon r$ to be decreases. For increasing bandwidth, height is to be increased whereas width, $\varepsilon r$, loss tangent is to be decreased.

\section{REFERENCES}

[1] Kumar G. and Ray K.P.,Broadband Microstrip Antenna,Artech House Publication,pp.1-291,2003.

[2] Kin Lu Wong, Compact and Broadband Microstrip Antennas,John Wiley \& Sons,pp.45-79,2002.

[3] S.S.Yavalkar, R.T.Dahatonde, S.S.Rathod, S.B.Deosarkar, "Comparative Analysis of Bandwidth Enhancement of Microstrip Patch Antenna using Various Geometries," IOSR Journal,Vol.3, Issue 4, Sep.2012 